\title{
FORMATION OF MICROPARTICLES INITIATED BY EXCIMER LASER PHOTOLYSIS OF Mo(CO)
}

\author{
A. V. EVSEEV, S. V. KAMAYEV, and R. H. SHARIPOV \\ Research Centre for Technological Lasers, Russia Academy of Sciences, \\ 140700 Shatura, Moscow Region, Russia
}

(Received 5 September, 1994)

\begin{abstract}
Formation of microparticles initiated by $\mathrm{XeCl}$ laser radiation photolysis of $\mathrm{Mo}(\mathrm{CO})_{6}$ mixed with buffer gas was studied. It was shown that micron-sized heavy objects (microparticles) of little mobility were formed and scattered the beam of the He-Ne probe laser. The microparticle mass and size were determined from probe laser beam scattering. The average density of microparticle was estimated $\left(\rho \approx 0.1 \mathrm{~g} \cdot \mathrm{cm}^{-3}\right)$.
\end{abstract}

KEY WORDS: Excimer laser, photolysis, transition metal hexacarbonyl.

\section{INTRODUCTION}

UV photolysis of gaseous transition metal carbonyls is the subject of intensive investigation. ${ }^{1-4}$ This process allows to obtain films of refractory metals on different substrates at room temperature ${ }^{5-8}$ and to initiate some chemical reaction. ${ }^{9,10}$ It was found recently that atomic and molecular clusters as well as microparticles are formed as a result of chromium, wolfram and molybdenum hexacarbonyl photolysis. ${ }^{11}$ This paper discusses the microparticle formation caused by $\mathrm{XeCl}$ laser beam excitation of $\mathrm{Mo}(\mathrm{CO})_{6}$ mixed with buffer gases. The goal of this paper is the determination of microparticle mass, characteristic size, and average density.

\section{EXPERIMENTAL}

The formation of micron-sized particles was registered by the He-Ne laser beam scattering $(\lambda=632.8 \mathrm{~nm})$. For the experimental determination of microparticle mass $(M)$, the velocity of microparticle fall in gravity field (V) and the diffusion coefficient (D) were measured. Mass is related to $\mathrm{V}$ and $\mathrm{D}$ in accordance with:

$$
M=(k T / g)(V / D) \text {, }
$$

where $\mathrm{k}$ is the Boltzmann constant, $\mathrm{g}$ is the free fall acceleration, $\mathrm{T}$ is absolute temperature. Indeed, for the settled fall velocity

$$
\mathrm{Mg}+\mathrm{f}_{\mathrm{r}}=0 \text {, }
$$

where $f_{r}(V)$ is the resistance force of medium to act on a particle moving in the buffer gas. According to ${ }^{12}$ the set fall velocity is described as

$$
\mathrm{V}=\mathrm{bMg} \text {, }
$$


where $b$ is the particle mobility related to the diffusion coefficient by Einstein relationship

$$
\mathrm{D}=\mathrm{bkT} \text {. }
$$

The combination (2) and (3) gives (1). According to ${ }^{12}$ the diffusion coefficient enables evaluating microparticle size (d), so for small amount diffusion of heavy spherical particles in light gas, we have:

$$
\mathrm{D}=0.6 \frac{(\mathrm{kT})^{3 / 2}}{\mathrm{~d}^{2} \mathrm{P}_{\mathrm{b}} \mathrm{m}_{\mathrm{b}}},
$$

where $P_{b}$ and $m_{b}$ are the pressure and the mass of the buffer gas molecules. Assuming spherical shape of microparticles, their average density is given as

$$
\rho=6 \mathrm{M} /\left(\pi \mathrm{d}^{3}\right) .
$$

Experimental setup is shown in Figure 1. $\mathrm{Mo}(\mathrm{CO})_{6}$ was excited by $\mathrm{XeCl}$ laser $(\lambda=308 \mathrm{~nm}$, pulse widths $10 \mathrm{~ns}$ FWHM). Laser beam was focused into the center of stainless steel chamber through quartz input windows. The beam cross-sectional area was $1.5 \times 0.4 \mathrm{~mm}^{2}$ by $\mathrm{e}^{-1}$ level in the constriction region, the caustic length was $\approx 1 \mathrm{~cm} .1 \mathrm{~mW}$ He-Ne laser beam of $0.3 \mathrm{~mm}$ in diameter was used as a probe one.

Exciting and probing beams in propagated parallel towards each other. The scattered light was registered normally to the propagation of laser beams through the side window of the chamber. It was focused to the vertically oriented input slit of the monochromator by a quartz lens. The monochromator was tuned to $\lambda=632,8 \mathrm{~nm}$. The scattered radiation was registered by the photomultiplier in photoncounting mode. The photomultiplier signal was amplified, discriminated and delivered to the PC-connected digital oscillograph through the $10 \mathrm{msec}$ time constant integrator. The residual pressure before leaking the gas in was not more than $10^{-5}$ Torr. The investigation was carried out at $\mathrm{Mo}(\mathrm{CO})_{6}$ pressure of $20-120 \mathrm{mTorr}$, the buffer gas pressure varied from 0 to 100 Torr. The scattering of the probe-beam was not observed in pure $\mathrm{Mo}(\mathrm{CO})_{6}$

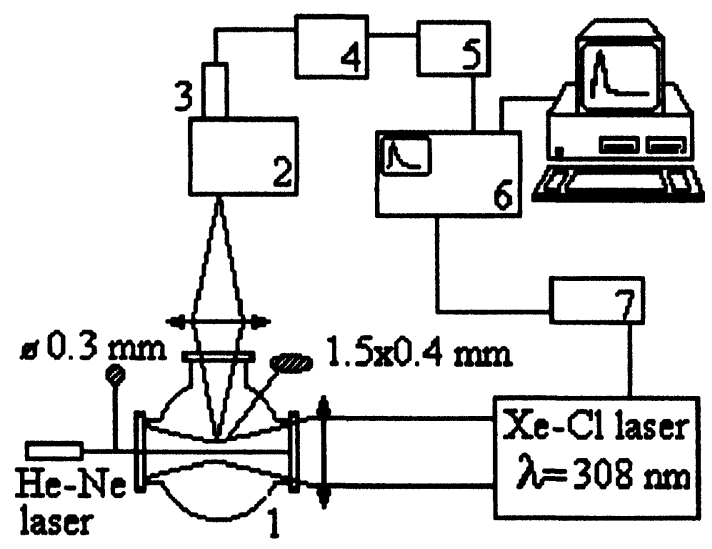

Figure 1 The scheme of the experiment. 1-vacuum chamber, 2-monochromator, 3-photomultiplier, 4-amplifier, 5-integrator, 6-digital oscillograph, 7-generator. 
for this experimental scheme. The buffer gas pressure threshold of scattering initiation depended on the laser pulse rate repetition and the type the buffer gas. The results shown below were obtained for single pulse irradiation of $\mathrm{Mo}(\mathrm{CO})_{6}$. In that case, the reliable results were obtained for the laser fluence of $60 \mathrm{~mJ} / \mathrm{cm}^{2}$ at He pressure of $\geqslant 3$ Torr.

\section{RESULTS AND DISCUSSION}

Oscillograms of the scattered radiation intensity $I_{\text {scat }}(t)$ for exciting and probing beams propagated coaxially are shown in Figure 2. The emission from the caustic region of the $\mathrm{XeCl}$ laser beam was not observed without $\mathrm{He}-\mathrm{Ne}$ laser beam. As He pressure and exciting laser fluence were increased, the risetime $I_{\text {scat }}(t)$ decreased but the signal amplitude grew. In order to determine the dimension of the region to scatter the probing beam effectively, the He-Ne laser beam was displaced in vertical plane parallel to the $\mathrm{XeCl}$ laser beam.

The signal amplitude dropped fast as the beam was displaced up (the inset at the upper part of Figure 3) and for the distance $\delta=1 \mathrm{~mm}$ the amplitude value was only several percent of the initial one. The duration of the signal risetime front did not change in this case.

The kinetics of the scattering was absolutely different when the beam was displaced down (Figure 3 ). As the distance $(\delta)$ was increased, the duration of scattered signal grew, and the maximum of $I_{\text {scat }}(t)$ was shifted to a longer time region. The kind of scattering kinetics indicated that the scattering region fell and expanded during the fall. As the position of maximum $\left(\mathrm{t}_{\max }\right) \mathrm{I}_{\text {scat }}(\mathrm{t})$ was linearly proportional to the distance $(\delta)$ (Figure 4), the fall velocity of this region was constant and, for example, can

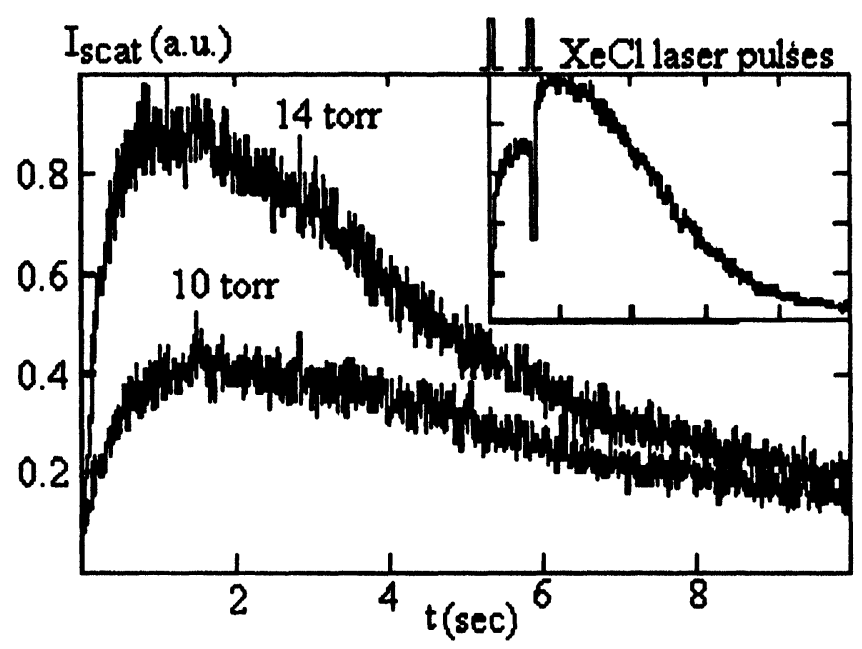

Figure 2 Dependence of scattering intensity vs time for coaxial laser beams propagation $(\delta=0)$. He pressure 10 and 14 Torr, $\Phi=70 \mathrm{~mJ} / \mathrm{cm}^{2}$. The time dependence $I_{\text {scat }}(t)$ in the case of $\mathrm{Mo}(\mathrm{CO})_{6}$ photolysis by two delayed pulses $(\Delta t=1 \mathrm{sec})$ is shown in the upper part of the figure. 


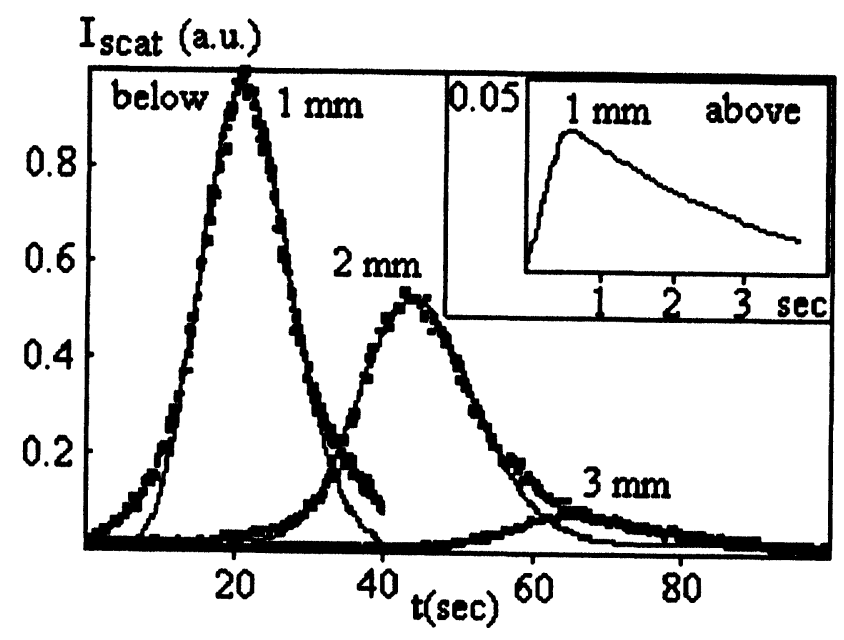

Figure 3 Dependence of scattering intensity vs time for various displacements of the He-Ne laser beam above (the insert at the upper part) and below axis of $\mathrm{XeCl}$ laser beam. He pressure 40 Torr, $\Phi=80 \mathrm{~mJ} / \mathrm{cm}^{2}$.

be evaluated as $2,1 \cdot 10^{-2} \mathrm{~cm} \cdot \mathrm{sec}^{-1}$ for $\mathrm{P}_{\mathrm{He}}=10$ Torr. The fall velocity decreased as $\mathrm{He}$ pressure increased $\left(\mathrm{V}=4,58 \cdot 10^{-3} \mathrm{~cm} \cdot \mathrm{sec}^{-1}\right.$ at $\mathrm{P}_{\mathrm{He}}=40$ Torr $)$ and increased as laser fluence increased. Assuming that fall velocity was constant and that when flying through the probe beam the size of the scattering region did not change significantly, we have for the vertical dimension: $V \cdot \Delta t$, where $V$ is the fall velocity, $\Delta t$ is the duration of the scattering signal. The evaluation showed that this dimension correlates to the exciting beam vertical dimension in the caustic region. Thus the region to scatter the probe beam was a compact formation which fell constantly and expanded slowly. The long duration of the scattering signal and the compactness of the scattering volume pointed to the low mobility of scattering objects and evidently to their stability

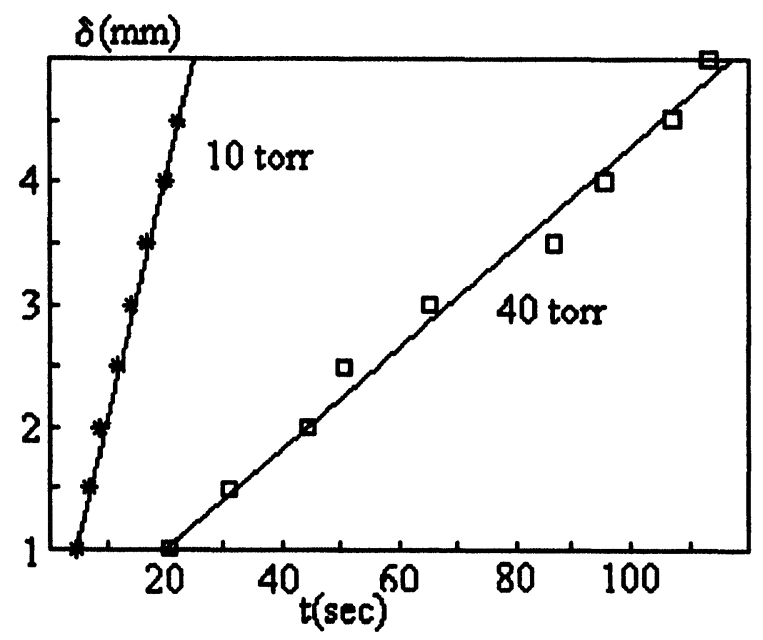

Figure 4 The dependence of maximum position $\left(t_{\max }\right)$ of the scattering signal on the displacement down at He pressure 10 and 40 Torr, $\Phi=80 \mathrm{~mJ} / \mathrm{cm}^{2}$. 
in a gas-phase. The increase of the scattering signal width can be caused by microparticle diffusion as well as by microparticle speed distribution. The microparticle diffusion in the external field is described by the Einstein-Fokker equation. ${ }^{13}$ Assuming Gaussian form of the initial microparticle concentration distribution and its axial symmetric we have:

$$
\mathrm{N}(\mathrm{r}, \mathrm{t}=0)=\mathrm{n}_{0} \exp \left(-\mathrm{r}^{2} / \mathrm{R}_{0}^{2}\right)
$$

For the case of homogenous external field the solution of the equation at the point of $\delta$ coordinate in the vertical plane is:

$$
\mathrm{N}(\mathrm{t}, \delta)=\frac{\mathrm{n}_{0} \mathrm{R}_{0}^{2}}{\mathrm{R}_{0}^{2}+4 \mathrm{Dt}} \exp \left[-\frac{(\delta-\mathrm{Vt})^{2}}{\mathrm{R}_{0}^{2}+4 \mathrm{Dt}}\right] .
$$

Where $\mathrm{n}_{0}$ is the microparticle concentration on the exciting beam axis, $r$ is the distance from the beam axis, $R_{0}$ is the half width of the initial distribution for $\mathrm{e}^{-1}$ level, $\delta$ is the displacement from the exciting beam axis to that of the probe beam, it is positive for down displacement and negative for up displacement, $\mathrm{V}$ is the microparticle drift velocity in the gravity field, $\mathrm{D}$ is the microparticle diffusion coefficient in the buffer gas. It was also supposed that all microparticles were identical and their collisions during the observation could be neglected. Assuming that the scattered beam radiation varies in proportion to the microparticle concentration, the kinetics of scattered radiation is given by (7). Obtaining $I_{\text {scat }}(t)$ for different $\delta$ values by (7), we can define $V$ and $\mathrm{D}$, as well as calculate mass, size and average density of microparticles using (1), (4) and (5). V, D and $R_{0}$ involved in (7) were evaluated originally in assumption that the size of microparticle "cloud" did not change significantly while crossing the probe beam. The velocity $\mathrm{V}$ was determined by the straight lines inclination (Figure 4$)$. The following method was used for the evaluation of $D$ and $R_{0}$ : the change in the initial distribution halfwidth (for $\mathrm{e}^{-1}$ level) in the system with the center of mass can be described as:

$$
\mathbf{R}^{2}=\mathbf{R}_{0}^{2}+4 \mathrm{Dt}
$$

Axially symmetric microparticle "cloud" of radius $\mathrm{R}$ crossed the probe beam in a time of $\Delta t=2 R / V$. Substituting $R=V(\Delta t / 2)$ in (8) we have the following expression

$$
(\Delta t / 2)^{2}=R_{0}^{2} / V^{2}+\left(4 D / V^{2}\right) t
$$

which relates $I_{\text {scat }}(t, \delta)$ width (for $e^{-1}$ level) to $R_{0}$ and $D$. $t_{\max }$ dependence of $(\Delta t / 2)^{2}$ is shown in Figure 5 for two He pressure values. $(\Delta t / 2)^{2}$ depends linearly on $t$. The extrapolation of these dependencies to $t=0$ allows to evaluate $R_{0}$ and their inclination enables evaluating of D. For dependencies given in Figure $5 R_{0}$ and $D$ are $0.5 \mathrm{~mm}$ and $1,9 \cdot 10^{-4} \mathrm{~cm}^{2} \cdot \mathrm{sec}^{-1}$ at $\mathrm{P}_{\mathrm{He}}=10$ Torr and $0,3 \mathrm{~mm}$ and $1,1 \cdot 10^{-5} \mathrm{~cm}^{2} \cdot \mathrm{sec}^{-1}$ at $\mathrm{P}_{\mathrm{He}}=40$ Torr respectively. V, $D$ and $R_{0}$ obtained by the same procedure were used as trial ones in order to compare $I_{\text {scat }}(t, \delta)$ with (7). As an example, time dependencies of microparticle concentrations in probe regions were calculated in accordance with (7) and are shown in solid lines in Figure 3. Because the microparticle "cloud" changed in size while flying through the probe beam, $I_{\text {scat }}(t, \delta)$ was asymmetric. The calculated dependencies reproduce positions of $\mathrm{I}_{\text {scat }}(\mathrm{t}, \delta)$ maximums and $\mathrm{I}_{\text {scat }}(\mathrm{t}, \delta)$ signal width well enough, but not the "tails" of $I_{\text {scat }}(t, \delta)$. 


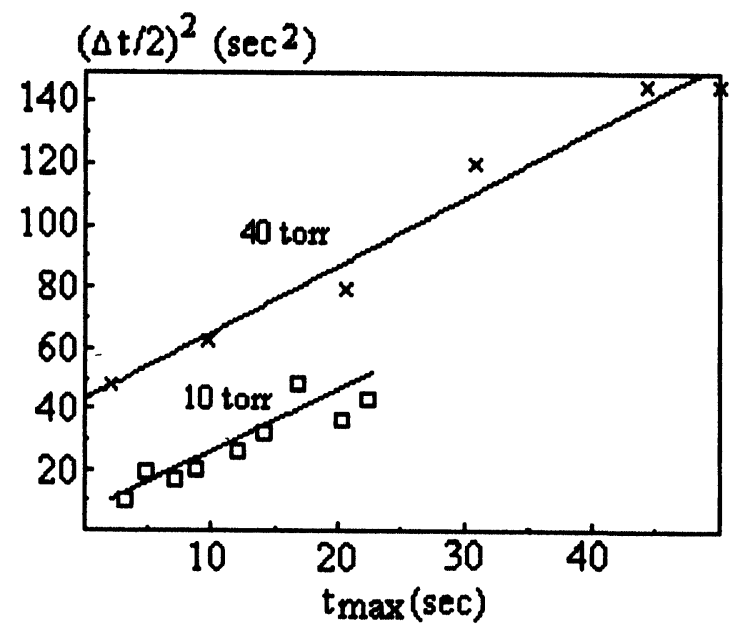

Figure 5 The $t_{\max }$ dependence of the $I_{\text {scat }}(t)$ halfwidth envelope (by $\mathrm{e}^{-1}$ level) squared by sequential displacement of the probe beam down at the distances $0-5 \mathrm{~mm}$ at He pressure 10 and 40 Torr, $\Phi=80 \mathrm{~mJ} / \mathrm{cm}^{2}$.

The difference between calculated and experimental dependencies can be explained first by the assumption of Gaussian axial symmetric initial distribution (6), and second by various drift velocities of microparticles because of the scatter in their parameters. The velocity distribution must have resulted in additional broadening of the $I_{\text {scat }}(t)$ envelope, in increasing of its asymmetry and in appearing of the "tails". Values $\mathrm{V}$ and $\mathrm{D}$ obtained from the comparison of $\mathrm{I}_{\text {scat }}(\mathrm{t}, \delta)$ with $(7)$ for different He and $\mathrm{Mo}(\mathrm{CO})_{6}$ pressures and different laser fluences $\Phi$ were used for the construction of microparticle mass and size plots. Mass and size of microparticles increased as $\mathrm{He}$ and $\mathrm{Mo}(\mathrm{CO})_{6}$ pressures and laser fluence grew. He pressure dependencies of the microparticles weight and size is shown in Figure 6. Under low He pressure and laser

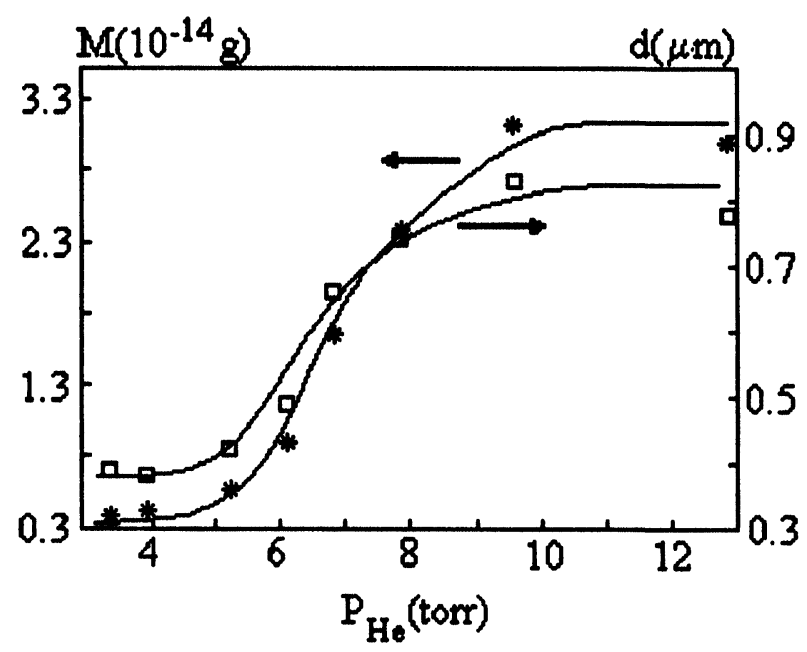

Figure 6 The He pressure dependence of microparticle weight $M$ and dimension d at $\Phi=80 \mathrm{~mJ} / \mathrm{cm}^{2}$. 
fluence, values $M$ and d grew fast as $P_{H e}$ and $\Phi$ were increased, in the case of $P_{H e}>10$ Torr and $\Phi>50 \mathrm{~mJ} \cdot \mathrm{cm}^{-2}$ microparticle parameters were only slightly affected by $\mathrm{P}_{\mathrm{He}}$ and $\Phi$, however, their concentration increased. For the dependencies shown in Figure 6 we have $\mathbf{M} \sim \mathrm{d}^{2.6}$. Since the exponent of the power approximates 3 , the microparticle substance density $\left(\rho \simeq 0.1 \mathrm{~g} \cdot \mathrm{cm}^{-3}\right)$ is only slightly dependent on $\mathrm{d}$. Low microparticle density, which differs significantly from those of solid $\mathrm{Mo}(\mathrm{CO})_{6}\left(\rho=1.96 \mathrm{~g} \cdot \mathrm{cm}^{-3}\right)$ and other Mo composition, points to the friable structure of microparticles. The fact that the exponent in the expression for $M(d)$ is not 3 can be explained by the fractal structure of microparticles. However, in order to obtain reliable accuracy in D and $\mathrm{M}$ evaluating, some additional studies are necessary.

\section{CONCLUSION}

We would like to discuss some special features of microparticle formation in $\mathrm{XeCl}$ laser beam photolysis of $\mathrm{Mo}(\mathrm{CO})_{6}$. The concentration rise time of microparticles which scatter He-Ne laser beam effectively is $<1 \mathrm{sec}$ and the size of the region where these microparticles are formed approximately equals the cross dimension of the exciting beam in the caustic region for $\mathrm{P}_{\mathrm{He}}>10$ Torr and $\Phi>50 \mathrm{~mJ} \cdot \mathrm{cm}^{-2}$. As $\mathrm{He}$ or $\mathrm{Mo}(\mathrm{CO})_{6}$ pressures and laser fluence decrease the rise time of microparticle concentration and the dimension of microparticle formation region increase. These results indicate that microparticle formation occurs due to the aggregation of the initial products. Really, the diffusion of initial products beyond the laser beam deduces their concentration. As a result, the time of formation of microparticles which scatter the probe beam effectively increases and so does the volume of their formation. For the volumes of formation in which the free path length of photolysis products between their collisions is comparable to the chamber size the formation of microparticles is not possible. We attribute the absence of the scattering signal in $\mathrm{Mo}(\mathrm{CO})_{6}$ photolysis without buffer gases to the rapid reducing of the concentration of photolysis products as a result of their leaving the caustic region of the $\mathrm{XeCl}$ laser beam because of its small size. We suppose that even in pure $\mathrm{Mo}(\mathrm{CO})_{6}$ photolysis there can appear some amount of microparticles to scatter the probe beam effectively if the size of the excited region is increased.

The most probable mechanism of microparticle formation is the condensation of photolysis products with vapor saturation pressure that is much lower than their partial pressure at room temperature. The addition of buffer gas first hinders the photolysis products divergence and second lowers their temperature. We should note that kind of initial products depends on the presence or absence of buffer gas. ${ }^{3,4}$ The formation of microparticles was also observed for such buffer gases as $\mathrm{Ar}, \mathrm{Kr}, \mathrm{Xe}, \mathrm{N}_{2}, \mathrm{O}_{2}$.

Because $I_{\text {scat }}(t)$ dependencies for different $\delta$ displacements and invariable $V$ and $D$ parameters are described satisfactorily by (7), we can conclude that microparticle parameters do not change significantly during their fall and therefore microparticles do not act on each other or on $\mathrm{Mo}(\mathrm{CO})_{6}$. The kinetics of $I_{\text {scat }}(t, \delta=0)$ for the case of $\mathrm{Mo}(\mathrm{CO})_{6} / \mathrm{He}$ mixture irradiated by two delayed pulses (the inset in the upper part of Figure 2) shows that microparticles formed in $\mathrm{Mo}(\mathrm{CO})_{6}$ photolysis can be easily fragmented by $\mathrm{XeCl}$ laser beam. 


\section{Acknowledgements}

We wish to thank Zherikhin A.N., and Panchenko V.Ya. for the discussion of our results and helpful comments.

\section{References}

1. M. Poliakoff, E.Weitz, Adv. Organomet. Chem., 25, 277 (1986).

2. T. R. Fletcher, R. N. Rosenfeld, J. Am. Chem. Soc., 107, 2203 (1985).

3. J. A. Ganske, R. N. Rosenfeld, J. Phys. Chem., 93, 1959 (1989).

4. V. N. Bagratashvili, A. V. Evseev, S. V. Kamayev, M. A. Markov, Chim. Phys., 9, 1023 (1990) (in Russian).

5. D. Bauerle, Appl. Phys. B., 46, 261 (1988).

6. R. Solanki, P. K. Boyer, G. J. Collins, Appl. Phys. Lett., 41, 1048 (1982).

7. D. K. Flynn, J. I. Steinfeld, J. Appl. Phys., 59, 3914 (1986).

8. V. N. Bagratashvili, A. V. Evseev, S. V. Kamayev, M. A. Markov, Proc. SPIE., 1352, 60 (1989).

9. K. J. Fu, E. R. Grant, J. Am. Chem. Soc., 104, 4270 (1982).

10. R. L. Wetten, K. J. Fu, E. A. Grant, J. Chem. Phys., 77, 3769 (1989).

11. A. V. Dem'yanenko, A. A. Puretzky, Spectrochimica Acta, 46A, 509 (1990).

12. E. M. Lifshiz, L. P. Pitayevsky, Physicskinetics, (in Russian), Moscow, Nauka (1976).

13. M. A. Leontovich, Introduction to thermodynamics. Statistical Physics., (in Russian), Moscow, Nauka (1983). 